\title{
Valoración económica de la vida del trabajador minero peruano*
}

\section{Economic Valuation of the Life of Peruvian Miners}

\author{
Jorge P. Mori Mojalott, ${ }^{* *}$ Jorge A. Alarcón-Novoa*** \\ Recibido: 2016-08-03 // Aprobado: 2016-10 - 12// Disponible en línea: 2017-01-30
}

Cómo citar este artículo: Mori Mojalott, J. P. y Alarcón-Novoa J. A. (2017). Valoración económica de la vida del trabajador minero peruano. Ambiente y Desarrollo, 21(40), 143-158. https://doi. org/10.11144/Javeriana.ayd21-40.vevt doi:10.11144/Javeriana.ayd21-40.vevt

\section{Resumen}

Se calcula el valor de la vida estadística (VVE) del trabajador minero peruano, año 2012, mediante la metodología de salarios hedónicos. El presente es el primer estudio de este tipo aplicado a un mercado laboral peruano. Se ha empleado la teoría de diferencias igualadoras, y se espera que mientras más elevado sea el riesgo de muerte en ocupaciones mineras, mayor será el salario asociado. La metodología ha requerido la definición previa de una ecuación de salarios explicados por características del trabajador y el puesto de trabajo. Se ha utilizado la Encuesta Nacional de Hogares de Perú del 2012 y estadísticas de muertes por accidentes laborales (MINEM). Con esta información se determinó la forma funcional de la ecuación salarial; se estimó el índice de riesgo mortal (0.0179). Finalmente, se obtuvo un VVE de US $\$ 978000$, valor superior al VVE promedio de US $\$ 654000$, obtenido por otros estudios para el Perú. Se espera que las conclusiones sirvan como primera aproximación al cálculo del beneficio por vidas salvadas en el sector minero, asociado a iniciativas gubernamentales que regulen la seguridad en el trabajo y el impacto ambiental de la actividad minera.

Palabras clave: valor de la vida estadística; preferencias reveladas; salarios hedónicos; transformación Box-Cox; índice de riesgo mortal

\footnotetext{
Este artículo se basa en la investigación de tesis de Jorge Pedro Mori Mojalott, titulada Valor de la vida. Estadística del trabajador minero en el Perú, para optar al título de economista de la Universidad Nacional Agraria La Molina (UNALM), Perú. La tesis fue dirigida por el Dr. Jorge A. Alarcón, coautor del artículo y sustentada en la UNALM en el 2015 y publicada en el 2016.

** Economista y Magíster en Finanzas, funcionario del Organismo Supervisor de la Inversión en Telecomunicaciones (Osiptel), Perú. Correo electrónico: jmorimojalott@gmail.com

*** Doctor en Economía Aplicada; profesor principal de la Facultad de Economía y Planificación, Universidad Nacional Agraria La Molina. Avenida La Molina s/n- La Molina, Apdo. 12-056- Lima 12, Perú. Correo electrónico: jalarcon@lamolina.edu.pe
} 


\begin{abstract}
We calculated the value of statistical life (VVE) of the Peruvian miner in 2012 using the hedonic wage methodology. This study is the first of its kind to be applied to a Peruvian labor market. We employed the equalizing differences theory and the expectation is that the higher the risk of death in mining activities, the higher the associated wage. The methodology required to define beforehand an equation of wages explained by the characteristics of the worker and the job. We also used the National Household Survey of Peru of 2012 and statistics of deaths due to occupational accidents (MINEM). With this information we determined the functional form of the wage equation and estimated the mortality risk index (0.0179). Finally, we came to a US $\$ 978,000 \mathrm{VVE}$, an amount greater than the US $\$ 654,000$ average VVE obtained by other studies for Peru. We expect the conclusions to serve as a first approach to the calculation of the benefit per lives saved in the mining sector, in association with government initiatives that regulate work safety and the environmental impact of mining activities.
\end{abstract}

Keywords: value of statistical life; revealed preferences; hedonic wages; Box-Cox transformation; mortality risk index 


\section{Introducción}

El valor de la vida estadística (VVE) es el valor de la disposición a aceptar (DAA) recursos con el fin de asumir una unidad adicional de riesgo mortal, o, alternativamente, la disposición a pagar (DAP) de los afectados por adoptar medidas de seguridad, a efectos de disminuir la probabilidad de sufrir algún tipo de daño o, incluso, la muerte.

En la actualidad no existe una valoración del riesgo de muerte por accidente laboral enfrentado por los trabajadores mineros peruanos. No obstante, este sector es preponderante en la economía peruana — solo en el año 2012, representaba el $12.17 \%$ del producto interior bruto (PIB), ${ }^{1}$ tercero en importancia, y alrededor del $60 \%$ de las exportaciones_, ${ }^{2}$ y sus trabajadores son los mejor remunerados. ${ }^{3}$ Sin embargo, existen importantes falencias en cuanto a aspectos de seguridad laboral, como se manifiesta en el elevado nivel de siniestralidad — solo en el ańo 2012 ocurrieron 52 accidentes fatales y entre el año 2009 y el 2013 la cifra fue de casi 55 fallecimientos en promedio por año—, ${ }^{4}$ situación que hace necesaria la estimación de un valor que refleje adecuadamente la valoración del riesgo enfrentado por estos trabajadores.

Esta valoración del riesgo se obtiene, precisamente, a través del cálculo del VVE, el cual resulta relevante en la aplicación de políticas públicas (Viscusi, 2003), debido a que permite valorar reducciones en el riesgo de mortalidad, aunque su uso está más difundido en la estimación de los beneficios por vidas salvadas. No obstante, en la evaluación de políticas de inversión en infraestructura y proyectos normativos del Gobierno peruano, que como consecuencia de su implementación generen la disminución del riesgo mortal enfrentado por los trabajadores, estos beneficios se encontrarían subvaluados, por cuanto no se emplea una medida adecuada para la evaluación de mejoras de la seguridad en el trabajo en el sector minero ni en otro sector productivo, y en cambio se opta por aproximaciones obtenidas desde estudios de otros países o, en el peor de los casos, este beneficio no se considera.

Por otro lado, el VVE suele ser empleado por organismos peruanos (p. ej., Osinergmin y OEFA $^{6}$ ) para el cálculo del componente del daño a la vida en multas por incumplimientos a normas aplicables a sectores productivos — sobre todo seguridad en el trabajo, salud ocupacional y medio ambiente-, que generen afectaciones a la vida y la salud de las personas. Sin embargo, estos cálculos no emplean el VVE específico del sector minero, aun cuando el incumplimiento se desarrolle en dicho sector; en su lugar se consideran homologaciones de valores recogidos de estudios aplicados a otros sectores productivos u otros países, principalmente desarrollados (p. ej., sector hidrocarburos). Respecto a lo precisado, la literatura señala que las conclusiones derivadas de estudios específicos no serían extensibles a otros escenarios, puesto que la elasticidad ingreso del VVE es positiva respecto al riesgo para la vida y la salud (Viscusi, 1993; Viscusi y Aldy, 2003; Viscusi, 2005).

Si bien existen variadas metodologías para el cálculo del VVE — como el enfoque del capital humano, el método de valoración contingente (preferencias declaradas), la técnica de transferencia de valores o el método de salarios hedónicos (preferencias reveladas)—, se considera que la metodología de salarios hedónicos es la forma natural de enfocar el tema (Thaler y Rosen, 1976), y es, en consecuencia, el método dominante en la literatura (Viscusi, 1993; Riera, Ripoll y Mateu, 2007; Viscusi y Aldy, 2003). Empero, esta metodología impide capturar todos los valores económicos que conforman la vida estadística, en cuyo caso autores como Vásquez (2006) consideran que el VVE calculado

$1 \quad$ Véase Banco Central de Reserva del Perú (BCRP, 2013).

2 Véase Banco Central de Reserva del Perú (BCRP, 2013).

3 Véase Instituto de ingenieros de minas del Perú (2011). Además, gran parte del salario percibido por el trabajador minero no se destina a cubrir sus necesidades básicas (alimentación, salud, vivienda, educación, etc.); estas son cubiertas por el empleador.

$4 \quad$ Véase Ministerio de Energía y Minas del Perú (MINEM, 2015).

5 Organismo peruano encargando de la supervisión de la inversión en energía y minería. Véase Vásquez (2006).

6 Organismo peruano encargado de la evaluación y fiscalización ambiental. 
mediante salarios hedónicos representa un valor intermedio entre las estimaciones del VVE obtenidas a través del enfoque del capital humano y el método de valoración contingente. ${ }^{7}$

Según lo señalado, en términos de la metodología de salarios hedónicos aplicada al sector minero, el VVE representa los incrementos en el salario derivados de incrementos en el riesgo mortal del trabajo minero. Así, este valor es el monto dinerario que un colectivo de trabajadores mineros aceptaría por admitir que un trabajador anónimo integrante de su grupo muera; es decir, representa la valoración que este colectivo particular de mineros peruanos asigna a una vida anónima o estadística.

En ese sentido, es objetivo del presente artículo calcular el VVE del trabajador minero peruano, como medida de los beneficios de iniciativas de gobierno que impliquen modificaciones en el riesgo mortal — precisamente en favor de la posibilidad de asumir riesgos humanos - y, asimismo, como una aproximación específica al valor del daño que se aplique en multas asociadas a incumplimientos de normas del sector minero. A este efecto, se plantea como hipótesis de trabajo que el VVE obtenido para el trabajador descrito, es superior al promedio y otros estimados individuales del VVE obtenido en otros estudios previos para el Perú (Miller, 2000; Vásquez, 2006; CISS, 2009), lo cual quiere decir que las estimaciones previas han estado subvaluadas.

Debe precisarse que el valor obtenido resulta directamente aplicable a la evaluación de proyectos públicos y normativos como los descritos y a incumplimientos sobre seguridad laboral en minería. En tanto que, debido a que no se consideran estadísticas sobre accidentes mortales derivados de afectaciones ambientales, no sería adecuada la aplicación directa del VVE en iniciativas públicas que regulen el impacto ambiental de la actividad minera. Sin embargo, las conclusiones podrían ser indirectamente aplicables y de manera referencial a estos escenarios.

Además de esta introducción, este artículo consta de cinco secciones más: los antecedentes, la sección metodológica, la presentación de los principales resultados encontrados, así como la discusión de estos y, finalmente, las conclusiones de rigor.

\section{Antecedentes $^{8}$}

El VVE se fundamenta en la teoría de las diferencias igualadoras, propuesta originalmente por Adam Smith quien argumentaba que los individuos son inducidos a tomar trabajos riesgosos mediante un set de diferenciales compensatorios. La teoría es mejor formalizada por Rosen (1974), en cuyo modelo se predice que trabajos con mayor riesgo mortal serán equilibrados con salarios más altos. Viscusi (2005) y Thaler y Rosen (1976) sostienen también que los individuos se ven inducidos a aceptar trabajos con determinado riesgo, a cambio de compensaciones en los salarios. Así, esta teoría predice que, en condiciones de competencia en el mercado de trabajo, dos empleos similares, excepto por el nivel de riesgo, tendrían que remunerarse de forma distinta (Albert y Malo, 1995).

La literatura referida al cálculo del VVE se ha desarrollado, principalmente, en países desarrollados, empleando en particular la metodología de salarios hedónicos. Por ejemplo, para los Estados Unidos, Viscusi (2003), mediante una especificación log-lineal de la ecuación de salarios y la consideración de un índice de riesgo mortal estructural, ${ }^{9}$ según ocupación y rama de actividad para un periodo de seis años (1992-1997), obtiene un VVE de US \$6.7 millones, en tanto que Aldy y Viscusi (2003), considerando

$7 \quad$ La valoración contingente es un método versátil, permite obtener valores de uso (directo e indirecto) y no uso, en forma separada o incluso de manera conjunta.

8 Todos los valores monetarios presentados en esta sección han sido llevados al ańo 2012. Para ello, se emplea el índice de precios al consumidor de cada país o economía analizada (zona euro), publicado por el Banco Mundial (2017). En tanto que, cuando ha sido necesaria la conversión de monedas, se ha empleado el tipo de cambio de soles (S/) por dólares (US\$) bancario venta, publicado por el BCRP, y el tipo de cambio euros por dólar publicado por la misma entidad.

9 Los autores consideran como índice estructural el índice de riesgo mortal de un periodo determinado. 
modelaciones del ciclo de vida en similar especificación de la ecuación de salarios, determinan un VVE que alcanza su tope a los veintinueve años, con un valor de US $\$ 8.4$ millones.

Dentro de la casuística europea, Albert y Malo (1995), quienes consideran índices de riesgo mortal y no mortal por ocupaciones, obtienen un VVE de US \$ 5.8 millones — todos los índices: accidentes mortales, no mortales y de enfermedades profesionales_y US \$5.9 millones — solo el índice de riesgo mortal - para el mercado laboral español. Una conclusión importante sería que la influencia de accidentes no mortales y enfermedades profesionales tendría un efecto marginal en el VVE; sin embargo, no incluirlos podría sesgar levemente hacia arriba al índice de riesgo mortal. Riera, Ripoll y Mateu (2007), por su parte, estimaron el VVE para el mismo mercado, considerando una especificación log-lineal e incluyendo índices específicos de riesgo mortal según ocupaciones y ramas de actividad. De esa manera, obtuvieron un VVE en el rango de US $\$ 2.5$ millones —índice de riesgo mortal puntual del año 2000 - y US $\$ 3.3$ millones —índice de riesgo estructural de un periodo de cuatro ańos: 1997-2000-.

En Latinoamérica las investigaciones que estiman el VVE son relativamente escasas (o poco difundidas); son también muy pocos los estudios que han usado la metodología de salarios hedónicos. En tal sentido, Parada, Riquelme y Vásquez (2013) emplearon el método de salarios hedónicos en Chile y estimaron el VVE en US \$12.8 millones. Anteriormente, Bowland y Beghin (1998) habían estimado el VVE asociado a la reducción de mortalidad debido a la contaminación del aire en Santiago de Chile, con el método de transferencia de beneficios de países desarrollados, y reportaron el VVE en un rango de US $\$ 0.85$ millones a US $\$ 1.1$ millones. Hammitt e Ibarrarán (2006) utilizaron salarios hedónicos y valoración contingente para estimar el VVE asociado a problemas de salud y mortalidad en la zona metropolitana de la Ciudad de México. Entrevistaron a mil personas y estimaron el VVE en un rango de US $\$ 176000$ a US $\$ 614000$ — los mayores obtenidos con valoración contingente y los más bajos con salarios hedónicos-. Márquez y Abella (2012) hicieron lo propio en Bogotá, para lo cual utilizaron el método de valoración contingente, y obtuvieron un VVE de US \$66000.

En lo que respecta a Perú, solo uno de los aportes consultados centra su análisis de manera específica en el VVE del país; los otros lo estiman como parte de un conjunto de valoraciones del VVE para países subdesarrollados. En todos los casos emplean la técnica de transferencia de valores, por lo que, si bien no utilizan directamente la metodología de salarios hedónicos, los estudios tomados de fuente sí lo hacen. Vásquez (2006), ${ }^{10}$ por ejemplo, desarrolla un estudio de aplicación a la fiscalización de empresas del sector peruano de hidrocarburos, con el objetivo de mejorar la capacidad sancionadora (multas) de Osinergmin. A tal efecto estima el VVE en US \$656000. En otros estudios, con varios países desarrollados y emergentes, Miller $(2000)^{11}$ y la Conferencia Interamericana de Seguridad Social (CISS) (2009) estimaron el VVE para Perú en valores de US \$542000 y US \$416000, respectivamente. En ambos casos el estimado VVE del Perú fue uno de los más bajos de la región.

De acuerdo con las anteriores consideraciones, el VVE promedio para el Perú, en el año 2012 y en dólares del mismo año, sería de, aproximadamente, US $\$ 654000$, valor que corresponde al promedio de cinco estimaciones - tres desde el estudio de Miller, una desde el estudio de Vásquez y una desde el aporte de CISS-.

Es importante añadir que, según los estudios revisados, el VVE para un peruano cualquiera sería de, aproximadamente, 145-150 veces la variable ingreso empleada en el cálculo — para los casos mencionados, el PIB per cápita-. Asimismo, teniendo en cuenta que la elasticidad ingreso del VVE es positiva (Viscusi, 2005, entre otros), se esperaría que en el caso de trabajadores mineros

10 Los estudios empleados son los presentados en Viscusi y Aldy (2003) y Viscusi (1993), desarrollados, fundamentalmente, mediante la metodología de salarios hedónicos.

11 Los autores calculan tres escenarios de VVE para el Perú: bajo (US\$ 451798), mejor (US\$ 542158 ) y alto (US\$ 1204795$)$. Para ello, emplean información de estudios aplicados, en particular, a mercados laborales de países desarrollados. 
—salarios superiores al de otras ocupaciones_, el VVE sea mayor que el presentado en la literatura aplicada al caso peruano.

Pese a lo que se ha señalado hasta aquí, debe tenerse en cuenta que, debido a la existencia general de mercados laborales no competitivos, ${ }^{12}$ la evidencia empírica no resulta contundente. Por ejemplo, los trabajos de Dorman y Hagstrom (1998) y Viscusi (2003) no mostraron diferenciales compensatorias por asumir mayores riesgos, más aun, los autores obtuvieron un VVE igual a cero o incluso negativo - en este último caso implicaría un menor pago por afrontar mayores riesgos. Según Viscusi (2003), tal vez porque los trabajadores no son conscientes del riesgo que enfrentan-. Sin embargo, los autores concluyen que la compensación salarial por el riesgo es plausible teóricamente, aunque menos cierta en la práctica.

\section{Metodología}

La metodología más aceptada para la determinación del VVE parte del enfoque de la disposición a pagar (DAP). ${ }^{13}$ Este enfoque se sustenta en el modelo teórico estándar de valoración del riesgo de mortalidad (Jones-Lee, 1974; Schelling, 1968; entre otros), el cual asume que la utilidad de un individuo racional está dada por la maximización del valor esperado de su utilidad, considerando dos escenarios y un único periodo (modelo estático). Los escenarios considerados son dos posibles estados de la naturaleza: sobrevivir con buena salud con una probabilidad asociada o, alternativamente, fallecer con una probabilidad de muerte. El individuo maximizará la siguiente función de utilidad esperada:

$$
E[U(p, w)]=(1-p) U_{v}(w)+p U_{m}(w)
$$

Donde: $w$ es el nivel de riqueza inicial del individuo, es la utilidad condicionada al estado de salud normal, $U_{m}(w)$ es la utilidad de la riqueza si el individuo fallece. Típicamente puede considerarse, desde una óptica altruista, como la utilidad que obtiene el individuo al dejar herencia.

En supuestos convencionales, se tiene en cuenta una disminución en el riesgo de muerte a cambio de una DAP por dicha disminución, manteniendo el nivel de utilidad — variación compensatoria a lo Hicks-. Luego de asumir el cambio en la probabilidad, se deriva la utilidad, y el resultado es la tasa marginal a la cual se intercambia riqueza por riesgo de morir, o el VVE.

$$
d w=\frac{\left(U_{v}(w)-U_{m}(w)\right.}{(1-\mathrm{p}) U_{v}^{\prime}(w)+p U_{m}^{\prime}(w)}=\mathrm{VVE}
$$

De lo anterior se deduce que el $V V E$ es igual a la tasa marginal de sustitución (TMS) entre riqueza y probabilidad de muerte (o, alternativamente, seguridad) (Jones Lee, 1992).

La estimación de la DAP puede realizarse mediante salarios hedónicos. Este método tiene la propiedad de estar microfundamentado, en cuanto no es sensible a indicadores (como lo es la tasa de descuento en la metodología de capital humano). Tampoco tiene el problema de las funciones casi siempre arbitrarias utilizadas en el modelo teórico estándar (como las desarrolladas por Jones-Lee, 1974, 1987; Viscusi, 1993; Carthy et al., 1999; Andersson y Treich, 2009, entre otros) y no involucra altos costos en tiempo, dinero y gran experiencia, como la requerida por el enfoque de valoración contingente.

La implementación de la metodología de salarios hedónicos requiere la especificación de una

12 La ecuación de salarios debe incluir, en lo posible, variables que reflejen el comportamiento no competitivo del mercado laboral, sin que ello implique invalidar la estimación del VVE.

13 Debe precisarse que para cambios marginales en salario y en el riesgo de muerte las DAA y las DAP son consideradas equivalentes. 
forma funcional de la ecuación de salarios — variable dependiente-, usualmente log-lineal, y de la consideración de las variables determinantes del salario — variables independientes-. Así, la ecuación de salarios de los trabajadores mineros peruanos puede especificarse a partir de un modelo de regresión lineal múltiple (MRLM).

Según los antecedentes, la mejor especificación corresponde a una forma funcional log-lineal de la ecuación de salarios. En ese sentido, para un trabajador “” la función hedónica es la siguiente:

$$
\ln \left(w_{j}\right)=\alpha_{0}+X_{j}^{\prime} \beta_{1}+Y j^{\prime} \beta_{2}+\gamma_{1} p_{j}+\gamma_{2} q_{j}+\gamma_{3} C N W_{j}+\mu_{j}
$$

Donde:

$w_{j}: \quad$ salario anual del trabajador

$\alpha_{0}: \quad$ intercepto del modelo

$X: \quad$ vector de variables de características individuales del trabajador “ $j$ ”, que determinan su salario

$Y: \quad$ vector de variables de características del puesto de trabajo, las cuales determinan el salario del trabajador $j$

$p_{j}: \quad$ índice de riesgo mortal

$q_{j}: \quad$ índice de riesgo no mortal

$C N W_{j}$ : compensaciones no salariales y no monetarias ${ }^{14}$ (del trabajador)

$\mu_{j}$ : error aleatorio, mide la influencia de otros factores estocásticos que determinan el salario del trabajador

$\beta_{1}, \beta_{2}, \gamma_{1}, \gamma_{2}, \gamma_{3}$ : son los parámetros que deben ser estimados en el análisis de regresión.

De acuerdo con la teoría de las diferencias igualadoras, el modelo de salarios hedónicos permite estimar el VVE a partir del valor de que "captura" el efecto del riesgo mortal en el salario de un trabajador minero, y dicho valor es una expresión de la disposición marginal a pagar de los trabajadores ante una reducción del riesgo de muerte (Riera, Ripoll y Mateu, 2007).

El logaritmo del salario anual del trabajador minero es la variable dependiente en la ecuación (3), y en este estudio se especifica de forma puntual (corte transversal 2012). El índice de riesgo mortal (IRM, $p_{j}$ ) es la variable regresora clave en el modelo de salarios hedónicos, pues su efecto en los salarios es la base para la estimación del VVE. El IRM se define como el número de muertes producidas en cada categoría ocupacional en un periodo determinado, dividido por el número de trabajadores en dicha categoría ocupacional en similar periodo, todo ello multiplicado por 10000 (unidades de $p_{j}$ ).

La tabla 1 muestra la fórmula para el cálculo de la DAP y el VVE a partir de la ecuación (3).

Tabla 1. Fórmula de cálculo para la DAP y el VVE

\begin{tabular}{cl}
\hline DAP & $D A P=\partial \mathrm{w}_{\mathrm{j}} / \partial p_{j}=\gamma_{j} w_{j}\left(\right.$ unidades de $\left.p_{j}\right)$ \\
\hline $\mathrm{VVE}$ & $V V E=\gamma_{i} \mathrm{w}^{*} 10.000$ \\
\hline
\end{tabular}

Fuente: elaboración propia

$14 \quad$ Algunos investigadores consideran la inclusión de las compensaciones representando aportaciones de la empresa por temas de salud o capacitaciones, entre otros (Riera, Ripoll y Mateu, 2007; Cobacho, López y Ramos, 2010). 
El procedimiento resumido para calcular el VVE del trabajador minero en el Perú ha sido el siguiente: (i) previamente se calculó el IRM por ocupación dentro de la actividad minera (número de fallecimientos ocurridos por accidentes laborales dividido por el número de trabajadores por categoría ocupacional, multiplicado por 10000) de forma estructural (periodo 2009-2013), según lo sugerido en los antecedentes; (ii) se especificó el modelo completo, considerando el salario representativo anual y todas las variables teóricamente importantes y empíricamente viables; (iii) se utilizó la transformación de Box-Cox aplicada a la variable dependiente (salario minero anual) para determinar la mejor forma funcional de la ecuación de salarios hedónicos, y el resultado fue una log-lineal; (iv) mediante mínimos cuadrados ordinarios (MCO), se estimaron los parámetros de regresión para dos modelos alternativos del logaritmo del salario, uno con el IRM puntual y otro con el IRM estructural, como variables explicativas; (v) por medio de los criterios de información de Akaike y de Schwarz (bayesiano), se procedió a seleccionar el modelo, para obtener luego el VVE.

\section{La transformación Box-Cox}

La evidencia empírica y la revisión teórica aconsejan el uso del método Box y Cox (1964) para aproximar la forma funcional más apropiada al comportamiento de los datos analizados. Consiste en la transformación de la variable dependiente mediante el uso de una familia de transformaciones paramétricas desde $y$ a $\gamma^{(\lambda)} . \lambda$ define la transformación particular según:

$$
y^{(\lambda)}= \begin{cases}\left(\mathrm{y}^{\lambda}-1\right) / \lambda, & \text { si } \lambda \neq 0 \\ \text { in } y, & \text { si } \lambda=0\end{cases}
$$

De este modo, para la transformación asociada conllevaría el uso del logaritmo de y, mientras que en el caso de $\lambda=1$, la transformación asociada sería la lineal. La aplicación de la técnica, respecto a la variable dependiente salario minero $\left(\mathrm{w}_{\mathrm{j}}\right)$, llevaría a la siguiente formulación de la ecuación hedónica (desde especificación general lineal):

$$
\left(\mathrm{w}_{\mathrm{j}} \lambda-1\right) / \lambda=\alpha+\mathrm{X}_{j}^{\prime} \beta_{1}+\mathrm{Y}_{j}^{\prime} \beta_{2}+\gamma_{1} p_{\mathrm{j}}+\gamma_{2} q_{\mathrm{j}}+\gamma_{3} c w_{\mathrm{j}}+\varepsilon_{\mathrm{j}}
$$

Donde $\alpha, \beta_{1}, \beta_{2}, \gamma_{1}, \gamma_{2}, \gamma_{3}$ son los parámetros estimados en el análisis de regresión y las variables regresoras son las mismas que en la ecuación (3). El enfoque presume que existe un tal que permite que el término de error se distribuya normalmente, sea homoscedástico y que el modelo cumpla con linealidad en los parámetros (Viscusi y Aldy, 2003). es estimado sujeto a la siguiente prueba: Ho: $\lambda=0$ versus $H a: \lambda \neq 0$.

Viscusi y Aldy (2003) refiere valores de 0.2 y 0.3. Por su parte Riera, Ripoll y Mateu (2007) estimaron un valor de $=0.2$ en un estudio aplicado al mercado laboral espańol. La evidencia empírica se orientaría hacia el modelo log-lineal.

\section{La muestra y los datos}

Para la estimación del VVE del trabajador minero en el Perú, así como la comprobación de hipótesis, en este estudio se utilizaron unidades de análisis correspondientes a individuos mayores de catorce años, con ocupación principal desarrollada en el sector minero, tanto metálico como no metálico, formal e informal, que trabajan por lo menos quince horas a la semana y habían declarado un sueldo y afrontado un riesgo de accidente laboral mortal mayor que cero. Por tanto, las conclusiones solo son aplicables a colectivos similares que afrontan el riesgo mortal señalado.

La mayor base de datos para "alimentación" del modelo, correspondiente a la minería metálica y no metálica, proviene de la Encuesta Nacional de Hogares de Perú del año 2012 (Enaho 2012). ${ }^{15}$ A

15 La Enaho 2012 es una encuesta que se realiza en el ámbito nacional, en el área urbana y rural, en los veinticuatro departamentos de Perú, más la provincia constitucional del Callao. En ella se encuentra información 
efectos de categorización, se han utilizado datos del IV Censo Nacional de Población y Vivienda llevado a cabo por el INEI (CPV-2007) en el año 2007. Las estadísticas del CPV-2007 se complementaron con reportes de accidentes laborales mortales (2009-2013) publicados por el MINEM, con el fin de construir el índice de riesgo mortal.

El tamańo de la muestra correspondiente al sector minero es la considerada en la Enaho 2012. Esta comprende 745 trabajadores, de los cuales el $8.6 \%$ son mujeres y el $91.4 \%$ son hombres. Considerando factores de expansión de la Enaho, los porcentajes fueron 7.6 y $92.4 \%$, respectivamente. Las ramas de actividad consideradas aplicables al sector minero están en función de la Clasificación Industrial Internacional Uniforme (CIIU) en su tercera revisión INEI (1010, 1310, 1320, 1410, 1422 y 1429). Después de la respectiva depuración, el tamaño final de la muestra usada fue de 522 individuos.

\section{Resultados y discusión}

En atención al hecho de que los datos corresponden a un modelo de corte transversal, el primer paso fue la construcción de la variable que expresa el riesgo de accidente mortal, el cual se diferencia según las ocupaciones en el sector minero. Sin embargo, debe recordarse que, debido a que las fatalidades son eventos "raros", autores como Viscusi (2003) consideran que es preferible construir y utilizar un índice de riesgo mortal estructural de un periodo como representativo de la muestra analizada. En ese sentido, considerando las conclusiones obtenidas de los criterios de información, resultó apropiado el uso de estadísticas de fatalidades de un periodo de cinco años y con la mayor cantidad de información disponible. El periodo considerado para la construcción del IRM fue del año 2009 al 2013. Debe precisarse que, si bien el artículo considera información del año 2012 para el conjunto de variables, en el caso específico del riesgo mortal consideramos apropiado incluir la información más reciente disponible al momento de la elaboración de la investigación. ${ }^{16}$

Debe precisarse que no ha sido posible la inclusión de la variable riesgo no mortal, por lo que es posible que los valores calculados se encuentren sobreestimados; sin embargo, siguiendo a Albert y Malo (1995), se espera que esta diferencia no sea significativa.

\section{Índice de riesgo mortal (IRM)}

El IRM para el periodo 2009-2013 se define como el número de fallecimientos por accidentes laborales promedio producidos en tal periodo por cada categoría ocupacional $(\overline{F G O})$, dividido por el número de trabajadores promedio en dicha categoría ocupacional $(\overline{T G O})^{17}$ para el periodo indicado, multiplicado por 10 000. Formalmente:

$$
(\overline{I R M})_{i, 2009-2013}=\left(\overline{F G O}_{\mathrm{i}, 2009-2013} \overline{T G O}_{i, 2009-2013}\right)(10000)
$$

Los cálculos de los índices de riesgo mortal se presentan en la tabla 2.

detallada sobre las características de los trabajadores y de sus empleos (modulo 5); sin embargo, no se encuentran variables como el nivel de satisfacción laboral, el nivel de responsabilidad del trabajador, entre otras, que son incluidas habitualmente en los modelos presentes en la literatura.

16 En la investigación original en la cual se basa el artículo, se realizó, adicionalmente, la estimación del VVE, considerando un IRM puntual del año 2012. Los resultados fueron levemente superiores a los aquí estimados.

17 Se considera que la proporción de trabajadores por categoría ocupacional se mantiene constante desde el CPV-2007. 
Tabla 2. IRM por categoría ocupacional de trabajadores mineros (2009-2013)

\begin{tabular}{|c|c|c|c|c|c|}
\hline Categoría ocupacional (1) & $A^{(2)}$ & $B^{(3)}$ & $C^{(4)}$ & $D^{(5)}$ & $I R M^{(6)}$ \\
\hline $\begin{array}{l}\text { 1. Miembros Poder Ejecutivo y } \\
\text { Legislativo, administración pública y } \\
\text { empresarial }\end{array}$ & $0.3 \%$ & 466 & 0 & $0 \%$ & 0.00 \\
\hline $\begin{array}{l}\text { 2. Profesionales, científicos e } \\
\text { intelectuales }\end{array}$ & $8.6 \%$ & 15095 & 2.4 & $4.4 \%$ & 1.59 \\
\hline $\begin{array}{l}\text { 3. Técnicos de nivel medio y trabajado- } \\
\text { res asimilados }\end{array}$ & $7.2 \%$ & 12682 & 1.8 & $3.3 \%$ & 1.42 \\
\hline 4. Jefes y empleados de oficina & $4.3 \%$ & 7637 & 0.0 & $0 \%$ & 0.00 \\
\hline $\begin{array}{l}\text { 5. Trabajadores de servicios personales y } \\
\text { vendedores del comercio y mercados }\end{array}$ & $0.6 \%$ & 1053 & 0.0 & $0 \%$ & 0.00 \\
\hline $\begin{array}{l}\text { 7. Obreros y operarios de minas, indus- } \\
\text { trias, y otros }\end{array}$ & $49.9 \%$ & 87960 & 21.8 & $40.1 \%$ & 2.48 \\
\hline $\begin{array}{l}\text { 8. Obreros de construcción, confeccio- } \\
\text { nes, papel, etc. }\end{array}$ & $6.3 \%$ & 11079 & 13.0 & $23.9 \%$ & 11.7 \\
\hline $\begin{array}{l}\text { 9. Trabajadores no calificados (servicios, } \\
\text { peones, vendedores ambulantes y afines) }\end{array}$ & $22.9 \%$ & 40419 & 15.4 & $28.3 \%$ & 3.81 \\
\hline Total & $100 \%$ & 176390 & 54 & & \\
\hline
\end{tabular}

(1) Categorías ocupacionales consideradas por el INEI en el CPV-2007 y en la Enaho, en función del CIUO88.

(2) Porcentaje de trabajadores mineros en cada categoría ocupacional según CPV-2007.

(3) Promedio anual de trabajadores sector minero: 176390 (MINEM).

(4) Número de fallecimientos por grupo ocupacional (MINEM).

(5) Porcentaje de trabajadores fallecidos en cada categoría ocupacional respecto al total de fallecidos.

(6) IRM en tantos por 10000 . Resulta del cociente: (C)/(B) multiplicado por 10000.

Fuente: elaboración propia

En promedio, en el periodo 2009-2013 ocurrieron 54 fatalidades por ańo. El grupo ocupacional 7 es el que más fatalidades concentra, $40.6 \%$ del total, en tanto que los grupos ocupacionales libres de riesgo fueron el 4 y el 5 .

\section{Uso de la transformación de Box-Cox}

Como se indica en la sección metodológica, la transformación de Box-Cox se basa en la modificación de la variable dependiente de modo tal que el modelo de regresión estimado mediante la ecuación (4) adopta, finalmente, la forma (3).

De la aplicación de la transformación de Box-Cox a la variable salario anual neto, y considerando que no ha sido posible la inclusión de la variable riesgo no mortal, se obtuvo un coeficiente. En tal sentido, Cameron y Trivedi (2009) consideran que el valor del coeficiente en el modelo de Box-Cox es difícil de interpretar y usar. Sostienen que si el valor calculado tiende hacia cero, existiría mucho más soporte hacia un modelo log-lineal como el finalmente empleado:

$$
\ln \left(w_{j}\right)=\alpha_{0}+X_{j}^{\prime} \beta_{1}+Y_{j}^{\prime} \beta_{2}+\gamma_{1} p_{j}+\gamma_{3} C N W_{j}+\mu_{j}
$$




\section{Evaluación de supuestos}

La ecuación (5) fue probada respecto a violación de supuestos. Se usó factores de inflación de varianzas y correlaciones simples para la determinación de no colinealidad entre las variables regresoras. Se utilizó el test de Ramsey (Reset) para corroborar la correcta especificación del modelo; no se encontraron variables regresoras importantes omitidas del modelo (95\% de confianza)..$^{18}$ Mediante la prueba de White, no se halló heteroscedasticidad. La presencia de sesgo de selección muestral (vinculado, en parte, a la correlación entre las explicativas y el error) no fue detectada mediante la aplicación del método bietápico de Heckman (1979).

\section{Presentación y análisis de resultados}

Los coeficientes, errores estándar y significancia estadística de las variables determinantes del salario minero, según la especificación salarial log-lineal, se muestran en la tabla 3.

Tabla 3. Resultado de la estimación de salarios hedónicos

\begin{tabular}{|c|c|c|c|c|c|}
\hline Denominación de variables/agregados & \multicolumn{2}{|c|}{ Dep: LOGSAL } & Coef. ${ }^{*}$ & $T$ & $(P>t)^{* *}$ \\
\hline & \multicolumn{2}{|l|}{ CONSTANTE } & 8.552 & 37.56 & 0.000 \\
\hline Expresa la edad del entrevistado & \multicolumn{2}{|l|}{ EDAD } & 0.006 & 2.95 & $0.003 \mathrm{a}$ \\
\hline $\begin{array}{l}\text { Indica el idioma del entrevistado. } \\
\text { Son dos variables ficticias; una es } \\
\text { "QUECHUA_AYM", la variable de } \\
\text { referencia es "ESPAÑOL". }\end{array}$ & \multicolumn{2}{|l|}{ QUECHUA_AYM } & 0.039 & 0.77 & $0.442 \mathrm{~d}$ \\
\hline \multirow[t]{8}{*}{$\begin{array}{l}\text { Grupo que incluye nueve variables } \\
\text { ficticias. Incluida en el modelo con } \\
\text { nueve variables categóricas: PRIM_- } \\
\text { COMP, SEC_INC, SEC_COMP, } \\
\text { TEC_INC, TEC_COMP, UNI_ } \\
\text { INC, UNI_COMP, POSTG (posgra- } \\
\text { do) y, como referente de comparación: } \\
\text { PRIM_INC (primaria incompleta) }\end{array}$} & \multicolumn{2}{|l|}{ PRIM_COMP } & 0.192 & 1.88 & $0.061 \mathrm{c}$ \\
\hline & SEC_INC & 0.196 & 1.93 & $0.055 \mathrm{c}$ & \\
\hline & SEC_COMP & 0.337 & 3.48 & $0.001 \mathrm{a}$ & \\
\hline & TEC_INC & 0.289 & 2.18 & $0.030 \mathrm{~b}$ & \\
\hline & TEC_COMP & 0.521 & 4.12 & $0.000 \mathrm{a}$ & \\
\hline & UNI_INC & 0.326 & 2.14 & $0.033 \mathrm{~b}$ & \\
\hline & UNI_COMP & 1.084 & 5.74 & $0.000 \mathrm{a}$ & \\
\hline & POSTG & 0.618 & 0.91 & $0.364 \mathrm{~d}$ & \\
\hline $\begin{array}{l}\text { Número de núcleos familiares en el } \\
\text { hogar }\end{array}$ & N_NUCLEO & & 0.084 & 1.78 & $0.075 c$ \\
\hline $\begin{array}{l}\text { Expresa la experiencia como poten- } \\
\text { cial factor determinante del salario. } \\
\text { Participa en el modelo como una } \\
\text { expresión cuadrática }\end{array}$ & EXPERIENCIA & & 0.029 & 3.21 & $0.001^{\mathrm{a}}$ \\
\hline EXPERIE & NCIA2 & -0.006 & -2.30 & $0.022 b$ & \\
\hline
\end{tabular}

18 Respecto a la no presencia de términos funcionales no lineales de las variables explicativas. 


\begin{tabular}{|c|c|c|c|c|}
\hline Denominación de variables/agregados & Dep: LOGSAL & Coef. $*$ & $T$ & $(P>t)^{* *}$ \\
\hline Contabilidad formal de la empresa & CONTAB_FORMAL & 0.264 & -2.87 & $0.004 \mathrm{a}$ \\
\hline $\begin{array}{l}\text { Es una variable que expresa el tipo de } \\
\text { empresa: SERVICE es una variable } \\
\text { ficticia y "EMP_PRIV" la alternativa }\end{array}$ & SERVICE & -0.117 & -1.79 & $0.075 \mathrm{c}$ \\
\hline $\begin{array}{l}\text { Expresa la modalidad de } \\
\text { contratación, compuesta } \\
\text { por cinco variables ficti- } \\
\text { cias: PLAZO_FIJO, A_- } \\
\text { PRUEBA, PRÁCTICAS } \\
\text { y SIN_CONTRATO, } \\
\text { Contrato INDEFINIDO } \\
\text { es la categoría de compa- } \\
\text { ración (no aparece) }\end{array}$ & PLAZO_FIJO & -0.052 & -0.68 & $0.495 \mathrm{~d}$ \\
\hline \multicolumn{2}{|c|}{ A PRUEBA } & -0.97 & $0.331 \mathrm{~d}$ & \\
\hline PRACTIC & -0.263 & -2.27 & $0.023 \mathrm{~b}$ & \\
\hline SIN_COI & NTRATO & -3.14 & $0.002 \mathrm{a}$ & \\
\hline $\begin{array}{l}\text { Representa el "ta- } \\
\text { maño" de la empre- } \\
\text { sa, según número de } \\
\text { trabajadores. Categorías: } \\
\text { DE_21_A_50_TRAB, } \\
\text { DE_51_A_100_TRAB } \\
\text { DE_101_A_500_TRAB, } \\
\text { MAS_500_TRAB } \\
\text { (mayor); la categoría } \\
\text { de comparación es: } \\
\text { "HASTA_20 trabajadores". }\end{array}$ & DE_21_A_50_TRAB & 0.059 & 0.55 & $0.580 \mathrm{~d}$ \\
\hline \multicolumn{2}{|c|}{ DE_51_A_100_TRAB } & 1.50 & $0.135 \mathrm{~d}$ & \\
\hline \multicolumn{2}{|c|}{ DE_101_A_500_TRAB } & 2.87 & $0.004 \mathrm{a}$ & \\
\hline \multicolumn{2}{|c|}{ MAS_500_TRAB } & 4.27 & $0.000 \mathrm{a}$ & \\
\hline $\begin{array}{l}\text { Cuantifica las horas laboradas por cada } \\
\text { trabajador, en su ocupación principal } \\
\text { en un año. Para el modelo se incluye a } \\
\text { todos aquellos trabajadores que laboran } \\
\text { más de quince horas a la semana }\end{array}$ & HORAS_AÑO & 0.000 & 1.74 & $0.083 \mathrm{c}$ \\
\hline $\begin{array}{l}\text { Recibimiento de compensaciones no } \\
\text { salariales }\end{array}$ & RECIBE_COMP & 0.155 & 2.45 & $0.015 b$ \\
\hline $\begin{array}{l}\text { Es el índice mortal. Señala la proba- } \\
\text { bilidad de fallecer por un accidente, } \\
\text { periodo 2009-2013. Para el modelo } \\
\text { se incluyen solo las ocupaciones con } \\
\text { algún riesgo }\end{array}$ & IRM2009_2013_CPV & 0.0179 & 2.57 & $0.011 b$ \\
\hline
\end{tabular}




\begin{tabular}{|c|c|c|c|c|}
\hline Denominación de variables/agregados & Dep: LOGSAL & Coef. ${ }^{*}$ & $T$ & $(P>t)^{* *}$ \\
\hline \multirow[t]{3}{*}{$\begin{array}{l}\text { Variable que categoriza las ocupaciones } \\
\text { en nueve grandes grupos. Se incluye en } \\
\text { el modelo mediante la variable "riesgo", } \\
\text { asociada a cada grupo ocupacional, } \\
\text { dado que las variaciones en el riesgo las } \\
\text { determinamos entre categorías ocupa- } \\
\text { cionales dentro del sector minero }\end{array}$} & GRUP_OCUP & -0.044 & -2.72 & $0.007 \mathrm{a}$ \\
\hline & Ajustado & 0.520 & $\begin{array}{l}\text { White } \\
\left(P>\chi^{2}\right)\end{array}$ & 0.11 \\
\hline & & 522 & $\begin{array}{l}\text { Ramsey } \\
\left(\mathrm{P}>\chi^{2}\right)\end{array}$ & 0.24 \\
\hline
\end{tabular}

* Son coeficientes corregidos según Wooldridge $(2014): \%(\Delta \mathrm{w}) \approx 100\left[\exp \left(\beta_{\mathrm{i}}\right) \Delta \mathrm{x}_{\mathrm{i}}-1\right]$

** Corresponden a la relación con errores estándar robustos de White.

a Significativa el $99 \%$. b Significativa al $95 \%$.

c Significativa al $90 \%$. d No significativa.

Fuente: elaboración propia

El coeficiente $\mathrm{R}^{2}$ ajustado es 0.52 ; se concluye que las variables independientes estarían explicando el $52 \%$ de las variaciones del logaritmo del salario. De la tabla 3 destacan los siguientes resultados:

- El coeficiente asociado al IRM resulta significativo al 95\%, con un valor de 0.0179 (tantos por $10000)$, lo cual indica que un incremento en el riesgo mortal de un punto (1/10 000) incrementaría el nivel salarial en $1.79 \%$, manteniendo los demás factores constantes. Esta situación evidenciaría la presencia de un diferencial compensatorio para enfrentar un mayor riesgo, hecho que validaría la teoría de las diferencias igualadoras.

- $\mathrm{Al}$ 99\% de confianza, existiría una relación positiva entre salario promedio y la experiencia laboral, lo cual indica que, a mayor experiencia, mayor es el nivel salarial, aunque la tasa de crecimiento no sería constante, dada la significancia del coeficiente de "EXPERIENCIA".

- Al 95\% de confianza, no existe suficiente evidencia para asegurar diferencias en el nivel salarial de los grupos con estudios relativamente bajos (primarios y secundarios) respecto al nivel sin estudios, pero sí entre este y los niveles superiores de estudios. Es esperable que el salario de una persona con estudios técnicos sea significativamente mayor que el salario medio de una persona sin formación (52.1 y $108.4 \%$ mayor en el caso de estudios técnicos y universitarios completos, respectivamente).

- Al mismo nivel de confianza, los trabajadores que perciben complementos no salariales obtienen un salario $15.5 \%$ más alto que aquellos que no los perciben.

\section{El valor de la vida estadística}

Si se emplea la fórmula del $V V E$ de la tabla 1 y lo obtenido en la tabla 3, y se considera que el valor del salario mediano para un trabajador minero promedio de todas las categorías ${ }^{19}$ es de S/ 14417 $(\bar{w})$, y dado el estimador del parámetro del índice de riesgo mortal de $0.0179\left(\hat{\gamma}_{j}\right)$, el VVE resulta en:

$$
\mathrm{VVE}=\hat{\gamma}_{\mathrm{j}} \bar{w}^{*}(10000) \rightarrow \mathrm{VVE}=\mathrm{S} / .2580643 \circ \$ 978186
$$

19 Debe recordarse que se incluye el salario mediano y no el promedio debido, a que la variable señalada resulta tener una distribución no normal, con lo cual muestra una gran sensibilidad respecto a valores extremos del salario. 


\section{Conclusiones}

El valor de la vida estadística (VVE) del trabajador minero peruano para el año 2012 resulta en US $\$ 978000$, lo que permite concluir que el VVE estimado es superior al promedio (US \$654000) y a los estimados individuales obtenidos en estudios previos; es decir, que las estimaciones previas aplicadas al sector minero habrían estado subvaluadas. Un motivo que es necesario considerar de por qué el valor calculado resulta superior a otras estimaciones realizadas para el Perú, es el hecho de que el nivel de ingresos en el sector minero sería superior al de otros sectores; un segundo motivo es el método de estimación (salarios hedónicos vs. transferencia de valores).

El valor calculado representa la DAA de los trabajadores mineros por aceptar el incremento del riesgo de accidente mortal laboral en una unidad (1/10000); es decir, aproxima la valoración que el colectivo de trabajadores mineros peruanos asigna al hecho de aceptar que alguno de sus integrantes muera a causa de un accidente de trabajo. Por tanto, puede emplearse en la evaluación de inversiones y proyectos normativos que modifiquen el riesgo de accidentes laborales; asimismo, el VVE puede emplearse como valor del componente del daño a los trabajadores del sector minero debido a incumplimientos a la normativa de seguridad en el trabajo.

Por otro lado, teniendo en cuenta que la información empleada no considera estadísticas sobre accidentes mortales derivados de afectaciones ambientales, se recomienda la aplicación indirecta o de forma referencial del VVE en aquellas iniciativas públicas que regulen el impacto ambiental de la actividad minera.

\section{Referencias}

Albert, C. y Malo, M. A. (1995). Diferencias salariales y valoración de la vida humana en España. Moneda y Crédito, (20), 87-125.

Aldy, J. E. y Viscusi, W. K. (2003). Age variations in workers' value of statistical life. Cambridge, Massachusetts: National Bureau of Economic Research, working paper 10199.

Andersson, H. y Treich, N. (2009). The value of a statistical life. Toulouse, Francia: Toulouse School of Economics (TSE), working paper series 09-015.

Banco Central de Reserva del Perú. (BCRP, 2013). Tablas anuales históricas del producto bruto interno por sectores productivos y tipo de cambio. Recuperado el 15 de diciembre del 2013, de http://www.bcrp.gob.pe/ estadisticas/cuadros-anuales-historicos.html

Banco Mundial. (2017). Indices de precios al consumidor, año base 2010, paises y años diversos. Recuperado el 30 de marzo del 2017, de http://datos.bancomundial.org/indicator/FP.CPI.TOTL

Bowland, B. y Beghin, J. (1998). Robust estimates of value of a statistical life for developing economies: an application to pollution and mortality in Santiago. Ames, Iowa: Center for Agricultural and Rural Development, Iowa State University, working paper 99-WP 214.

Box, G. y Cox, D. (1964). An analysis of transformations. Journal of the Royal Statistical Society. Series B (Methodological) 26(2), 211-252.

Cameron, A. y Trivedi, P. (2009). Microeconometrics using Stata. s. 1.: Stata Press.

Carthy, T., Chilton, S., Covey, J., Hopkins, L., Jones-Lee, M., Loomes, G., Pigdeon, N. y Spencer, A. (1999). On the contingent valuation of safety and the safety of contingent valuation: Part 2 - The CV/SG chained approach. Journal of Risk and Uncertainty, 17(3), 187-213. 
Cobacho, B., López, A. y Ramos, J. (2010). El coste de mortalidad asociado al consumo de tabaco en Espańa. Salud Pública, 84(3), 271-280.

Conferencia Interamericana de Seguridad Social. (CISS, 2009). Preferencias, gasto en salud y el valor de la vida estadística en América. México D. F.: CISS, documento de trabajo CISS/W P/09/01.

Dorman, P. y Hagstrom, P. (1998). Wage compensation for dangerous work revisited. Industrialy Labor Relations Review, 52(1), 116-135.

Hammitt, J. e Ibarrarán, M. (2006). The economic value of reducing fatal and non-fatal occupational risks in Mexico City using actuarial- and perceived-risk estimates. Health Economics Letters, (15), 1329-1335.

Heckman, J. (1979). Sample selection bias as a specification error. Econométrica, 47(1), 153-161.

Instituto de Ingenieros de Minas del Perú. (2011). Minería peruana: contribución al desarrollo económico y social. Lima: Área Difusión e Información Minera, N.o 5.

Instituto Nacional de Estadística e Informática del Perú. (INEI, 2007). Censo Nacional 2007: XI de población y VI de vivienda. Recuperado el 10 de diciembre del 2013, de https://www.inei.gob.pe/bases-de-datos/ Instituto Nacional de Estadística e Informática del Perú. (INEI, 2012). Encuesta Nacional de Hogares del año 2012. Recuperado el 10 de diciembre del 2013, de https://www.inei.gob.pe/bases-de-datos/

Jones-Lee, M. (1974). The value of changes in the probability of death or injury. Journal of Political Economy, 82(4), 835-849.

Jones-Lee, M. (1987). The economic value of life: a comment. Económica, 54(215), 397-400.

Jones-Lee, M. (1992). Paternalistic altruism and the value of statistical life. The Economic Journal, 102(410), 80-90.

Márquez, L. y Avella-Arévalo, H. (2012). Estimación del valor estadístico de la vida, asociado a la seguridad vial en Bogotá. Revista Ingenierías de la Universidad de Medellin, 11(21), 101-112.

Miller, T. (2000). Variations between countries in values of statistical life. Journal of Transport Economy and Policy, 34(2), 169-188.

Ministerio de Energía y Minas. (MINEM, 2009). Anuario minero. Lima: autor.

Ministerio de Energía y Minas. (MINEM, 2010). Anuario minero. Lima: autor.

Ministerio de Energía y Minas. (MINEM, 2011). Anuario minero. Lima: autor.

Ministerio de Energía y Minas. (MINEM, 2012). Anuario minero. Lima: autor.

Ministerio de Energía y Minas. (MINEM, 2013). Anuario minero. Lima: autor.

Ministerio de Energía y Minas. (MINEM, 2015). Estadísticas de accidentes fatales 2009-2013. Recuperado el 8 de diciembre del 2013, de http://www.minem.gob.pe/_publicaciones.php

Parada, M., Riquelme, J. y Vásquez, F. (2013). The value of a statistical life in Chile. Empirical Economics, 45(3),1073-1087.

Riera, A., Ripoll, A. y Mateu, J. (2007). Estimación del valor estadístico de la vida en España: Una aplicación del método de salarios hedónicos. Revista de Economía Pública, 181(2), 29-48.

Rosen, S. (1974). Hedonic prices and implicit markets: Product differentiation in pure competition. The Journal of Political Economy, 82(1), 34-55.

Schelling, T. (1968). The life you save may be your own. En S. B. Chase, Jr. (Ed.), Problems in public expenditure analysis (pp. 127-176). Washington, D. C.: Brookings Institution.

Vásquez, A. (2006). El valor de la vida estadística y sus aplicaciones a la fiscalización de la industria de hidrocarburos. Lima: Osinergmin, documento de trabajo n.o 18. 
Viscusi, K. (1993). The value of risks to life and health. Journal of Economic Literature, 31(4), 1912-1946.

Viscusi, K. (2003). The value of life: estimates with risks by occupation and industry. Cambridge, Massachusetts: Harvard Olin Center for Law, Economics and Business, discussion paper n.o 422.

Viscusi, K. (2005). The value of life. Cambridge, Massachusetts: Harvard Olin Center for Law, Economics and Business, discussion paper n. ${ }^{\circ} 517$.

Viscusi, W. K. y Aldy, J. E. (2003). The value of statistical life: a critical review of market estimates throughout the world. Journal of Risk and Uncertainty, 27(1), 5-76.

Thaler, R. y Rosen, S. (1976). The value of saving a life: evidence from the labor market. En N. Terleckyj (Ed.). Household production and consumption (pp. 265-302). New York: Columbia University Press.

Wooldridge, J. (2014). Introducción a la econometría (5.a ed.). México D. F.: Cengage Learning. 